\title{
Effect of providing pellets enriched with spores of parasiticide fungi to grazing cattle on the dynamics of faecal trematode egg-output
}

Cristiana Filipa Cazapal-Monteiro[a]" Fabián Leonardo Arroyo[ ${ }^{[a]}$, José Ángel Hernández ${ }^{[a]}$, Diana Freiría, Antonio Palomero ${ }^{[a]}$, María Isabel Silva, Jaime Sanchís ${ }^{[b]}$, Adolfo Paz-Silva ${ }^{[a]}$, Rita Sánchez-Andrade ${ }^{[a]}$, María Sol Arias ${ }^{[a]}$

[a] COPAR Research Group, Facultad de Veterinaria, Universidade de Santiago de Compostela (USC), Santiago de Compostela, Galicia, Spain

${ }^{[b]}$ Facultad de Veterinaria, Universidad de la República del Uruguay (UDELAr), Salto, Uruguay

${ }^{*}$ Corresponding author

e-mail: cristiana.cazapal@usc.es

\section{Abstract}

Ruminants maintained under pasturing management in humid areas can be at risk of infection by trematodes affecting the liver (Fasciola hepatica) or the rumen (Calicophoron daubneyi). Despite efficient anthelmintics are commercially available, the existence of encysted infective stages (metacercariae) on the vegetation enhances their infection. Metacercariae originate from cercariae, which exit off aquatic snails belonging to the Lymnaea genus, previously infected by a ciliated embryo developed inside the egg of the aforementioned trematodes. By considering that infected ruminants pass eggs by faeces, a strategy consisting of providing pellets with spores of parasiticide fungi to grazing heifers has been assayed. Spores of the ovicidal fungus Mucor circinelloides and the larvicidal fungus Duddingtonia flagrans were added during the mixing phase of manufacturing of a commercial formulation (DL Novillas $18^{\circledR}$, NANTA, Nutreco, Outeiro de Rei, Spain). Three groups of 7 grazing Friesian heifers infected by the gastric fluke C. daubneyi grazed during a 2-year period. Group G-F was daily supplemented with pelleted feed $+1.2 \times 106$ spores/kg feedstuff, and G-T and G-C were given concentrate without spores. Heifers in G-F and G-T received an oral dose of closantel (10 mg / kg bw Endoex, SP Veterinaria, Spain). Faecal samples were individually collected and analysed by means of the sedimentation test. Prior to the deworming, all the heifers shed eggs of the gastric fluke C. daubneyi in the faeces. One month after the administration of the anthelmintic, the analyses of the faeces collected in the G-F were negative. In the faecal samples of the heifers of G-T (dewormed and feeding on pellets without spores), eggs of the trematode were detected again from the 2nd month after treatment, and values ranging from 50 to 100 eggs per gram of faeces (EPG) were recorded throughout the study. The kinetics of $C$. daubneyi eggs reduced along the assay. Deworming of pasturing ruminants provides a solution against the parasites inside 
the hosts, but the existence of infective stages in the soil (herbage, water) constitutes a risk of infection. By feeding heifers with pelleted feed containing spores of parasiticide fungi, the eggs of the trematode $C$. dabneyi are exposed to an antagonistic effect responsible of delaying their development or destroying them. Manufacturing of concentrate with spores provides a very useful tool for spreading the fungal spores onto the environment where grazing heifers are feeding on. It is strongly recommended the administration of pelleted feed with fungi spores to heifers under pasturing regimes from humid areas.

Economical support: Research Project CTM2015-65954-R (Ministerio de Economía y Competitividad, Spain; FEDER). Dr. CF Cazapal-Monteiro is recipient of a fellowship from the Xunta de Galicia - Consellería de Educación e Ordenación Universitaria (Spain). 